\title{
Estabilización de la luxación inveterada de la quinta articulación carpo-metacarpiana mediante tenosuspensión con banda de extensor cubital del carpo
}

\section{Partial Slip-Extensor Carpi Ulnaris Tenosuspension Procedure for Stabilization of Old Dislocation of the Fifth Carpometacarpal Joint}

\author{
1 Unidad de Cirugía de Mano y Miembro Superior del Servicio de \\ Cirugía Ortopédica y Traumatología del Hospital Universitario "San \\ Cecilio" de Granada, Granada, España \\ ${ }^{2}$ Departamento de Cirugía y sus Especialidades, Facultad de \\ Medicina, Universidad de Granada, Granada, España \\ 3 Instituto de Investigación Biosanitaria de Granada (IBS), Granada, España \\ Rev Iberam Cir Mano 2018;46:135-142.
}

Pedro Hernández-Cortés ${ }^{1,2,3}$ Laura Gallegos Rodríguez ${ }^{1} \quad$ María Isabel Almagro Gil ${ }^{1}$

Address for correspondence Pedro Hernández-Cortés, MD, Departamento de Cirugía, Facultad de Medicina, Universidad de Granada, Avenida de la Investigación, 11. Granada 18016, España (e-mail: phc@ugr.es).

\section{Resumen}

Palabras Clave

- articulación carpometacarpiana

- luxación inveterada

- tenosuspension
Las fracturas luxaciones de la articulación carpometacarpiana (CMC) del $5^{\circ}$ dedo pasan desapercibidas con frecuencia. El diagnóstico y tratamiento tardíos conlleva con frecuencia malos resultados funcionales por dolor, pérdida de fuerza de prensión y el desarrollo de artrosis CMC. El tratamiento de las luxaciones inveteradas es un reto. Se han propuesto numerosas técnicas quirúrgicas al respecto. Presentamos una modificación de una tenoplastia de supensión, utilizando una banda del tendón del Extensor Carpi Ulnaris. Creemos que esa técnica tiene varias ventajas sobre otros procedimientos paliativos. En primer lugar, se preserva la movilidad del $5^{\circ}$ metacarpiano que se estabiliza sólo por ligamentoplastia al $4^{\circ}$ metacarpiano. En segundo lugar, mantiene la longitud del $5^{\circ}$ radio. En tercer lugar, admite una variante en la que la realización de túneles óseos en $4^{\circ}$ y $5^{\circ}$ metacarpiano favorece una mejor alineación, evitando la disrotación del $5^{\circ}$ dedo. Pensamos que esa técnica es el mejor tratamiento en las luxaciones inveteradas de la $5^{a}$ articulación CMC.

Fracture-dislocations of the little finger carpometacarpal (CMC) joint are commonly missed. Delayed diagnosis and treatment will usually result in an undesirable outcome of pain, reduced grip strength, and degenerative arthritis. Treatment of the old dislocations of the fifth carpometacarpal joint is challenging. Numerous surgical techniques have been proposed. Here, we present a modification of a CMC joint suspensionplasty, using a partial slip of the Extensor Carpi Ulnaris tendon. We believe this technique has several advantages compared with other palliative surgical procedures. First, mobility of the fifth metacarpal is greatly preserved because the fifth metacarpal is stabilized only by the ligament substitute against the fourth metacarpal. Second, the length of the fifth ray can be maintained effectively. Third, malrotation of the little finger can be prevented by drilling the holes of the fifth and fourth metacarpals in the proper direction. We believe this surgical technique could be a better treatment option for old dislocation of the fifth CMC joint. received

July 22, 2018

accepted

October 1, 2018

published online

November 5, 2018
DOI https://doi.org/

10.1055/s-0038-1675563. ISSN 1698-8396.
Copyright @ $\odot 2018$ Thieme Revinter

Publicações Ltda, Rio de Janeiro, Brazil
License terms

(ㄷ) (i) $\ominus$ (5) 


\section{Introducción}

Las fracturas-luxaciones hamatometacarpianas (HMTC), abarcan un amplio rango de lesiones complejas que incluyen las luxaciones carpo-metacarpianas (CMC) puras, palmares o dorsales, aisladas del $5^{\circ}$ metacarpiano o combinadas del $4^{\circ}$ y $5^{\circ}$ y las fracturas-luxaciones que pueden interesar a la base de los metacarpianos $\mathrm{y} / \mathrm{o}$ el hueso ganchoso. ${ }^{1}$

Precisamente, Kim y Shin $^{2}$ proponen una clasificación según la afectación articular del ganchoso y la base del metacarpiano, estableciendo la lesión tipo I (luxación simple), tipo IIA (luxación con fractura intraarticular de base $4^{\circ}$ MTC), IIB (luxación con fractura de dorso de ganchoso menor de $1 / 3$ de la superficie articular) y tipo III (luxación con fractura de dorso de ganchoso mayor de $1 / 3$ de superficie articular).

Las fracturas luxaciones CMC son infrecuentes, suponen menos del $1 \%$ de todas las lesiones en la mano y muñeca, ${ }^{3}$ aunque su verdadera incidencia es desconocida e infraestimada porque pasan con frecuencia desapercibidas, debido al bajo índice de sospecha del médico de urgencias en ese tipo de lesión, la inflamación de partes blandas que obstaculiza la localización del área dañada, o la superposición de los metacarpianos y huesos del carpo en la radiografía, que dificultan la interpretación. ${ }^{4}$ Por ello, el diagnóstico y el tratamiento se demora en muchas luxaciones.

La reducción cerrada suele fracasar en los pacientes diagnosticados tardíamente y a menudo requieren cirugía abierta para asegurar una reducción adecuada..$^{5}$ Aunque la discapacidad y artritis CMC en las reducciones subóptimas es menos frecuente de lo que cabría esperar, ${ }^{6}$ las fracturas luxaciones groseramente desplazadas son causa de dolor, deformidad e interfieren negativamente con la función de prensión.

Para los pacientes con luxaciones inveteradas y aquellos en fase de secuelas, se han descrito diversos procedimientos quirúrgicos paliativos que incluyen artrodesis hamatometacarpiana, artrodesis intermetacarpiana, artroplastia de resección o artroplastia de interposición con tendón autólogo o implante de silicona. ${ }^{7-10}$ Kato y Fukumoto $^{11}$ proponen en 2016, una artroplastia de resección-tenosuspensión con una banda del tendón del Extensor Carpi Ulnaris (ECU) para tratar las secuelas de las fracturas luxaciones $\mathrm{CMC}$ del $5^{\circ}$ radio de la mano, aplicando la misma filosofía de la trapeciectomía y tenosuspensión que se realiza para el tratamiento de la rizartrosis.

Nosotros hemos utilizado la tenosuspensión con parte del tendón del ECU para la estabilización de luxaciones CMC del $5^{\circ}$ metacarpiano inveteradas, sin la resección de la base del metacarpiano, con buenos resultados y exponemos en este trabajo la sistemática de cómo la realizamos.

\section{Indicaciones}

Luxaciones CMC del $5^{\circ}$ metacarpiano, agudas o inveteradas, que no se puedan reducir y fijar por métodos cerrados. La indicación se puede ampliar también a las luxaciones CMC de $4^{\circ}$ y $5^{\circ}$ radio, modificando la técnica, y puede plantearse como gesto complementario en las luxaciones con fractura del ganchoso susceptibles de reducción abierta y osteosíntesis.

\section{Contraindicaciones}

El procedimiento no debe realizarse si a la luxación CMC del $5^{\circ}$ radio se asocia una fractura del tercio proximal del $4^{\circ}$ metacarpiano.

La buena evolución en la mayoría de los casos de la reducción cerrada y fijación percutánea, no hace rentable el procedimiento que se describe a continuación en las luxaciones agudas.

\section{Anatomía Quirúrgica}

La articulación HMTC es una articulación en silla de montar. La base convexa del $5^{\circ}$ metacarpiano se aloja en la concavidad de la carilla articular del ganchoso. ${ }^{12}$ Ambos huesos tienen una carilla articular plana adicional para articular con el $4^{\circ}$ metacarpiano.

Esta articulación tiene una inclinación radio-ulnar en el plano frontal para permitir $10-20^{\circ}$ de supinación, que favorece la inestabilidad y mayor incidencia de luxación de la articulación CMC del $5^{\circ}$ radio por deslizamiento en dirección ulnar bajo la tracción del tendón del ECU que se inserta en la base del $5^{\circ}$ metacarpiano, ${ }^{13}$ como ocurre en las fracturas luxaciones del $1^{\circ}$ metacarpiano bajo la tracción del tendón del abductor largo del pulgar. La similitud ha licitado la denominación de "Benett del $5^{\circ}$ metacarpiano".

La articulación tiene fuertes estructuras ligamentosas en sus aspectos palmar, dorsal e interóseo. El ligamento interóseo proporciona una robusta unión entre $4^{\circ}$ y $5^{\circ}$ metacarpianos y hace que ellos se comporten como una unidad, siendo el más importante para la estabilidad, y es el elemento que se pretende suplir con la técnica que presentamos. El Gancho del ganchoso es un contrafuerte frente a luxaciones volares.

El abordaje quirúrgico que se propone pone en riesgo alguna de las ramas nerviosas dependientes de la rama cutáneo dorsal del nervio cubital y se recomienda que sea identificada y protegida durante la cirugía, para evitar su lesión y el desarrollo de un neuroma doloroso.

La articulación HMTC se encuentra inmediatamente distal al retináculo extensor, pero conviene realizar una apertura parcial del mismo para proporcionar una mejor exposición y facilitar la extracción del tendón para la plastia.

El tendón extensor propio del $5^{\circ}$ dedo cruza el campo quirúrgico y debe desplazarse en algunos momentos a radial y en otros, en sentido cubital, para no interferir con la visualización o el desarrollo de la técnica.

Por último, advertimos que la toma de una banda del tendón del ECU, ha de realizarse sin la separación del mismo del cubito distal, para evitar una luxación del mismo en el postoperatorio.

\section{Técnica Quirúrgica}

En el estudio preoperatorio, realizamos un TAC para identificar y caracterizar una posible fractura del hueso 

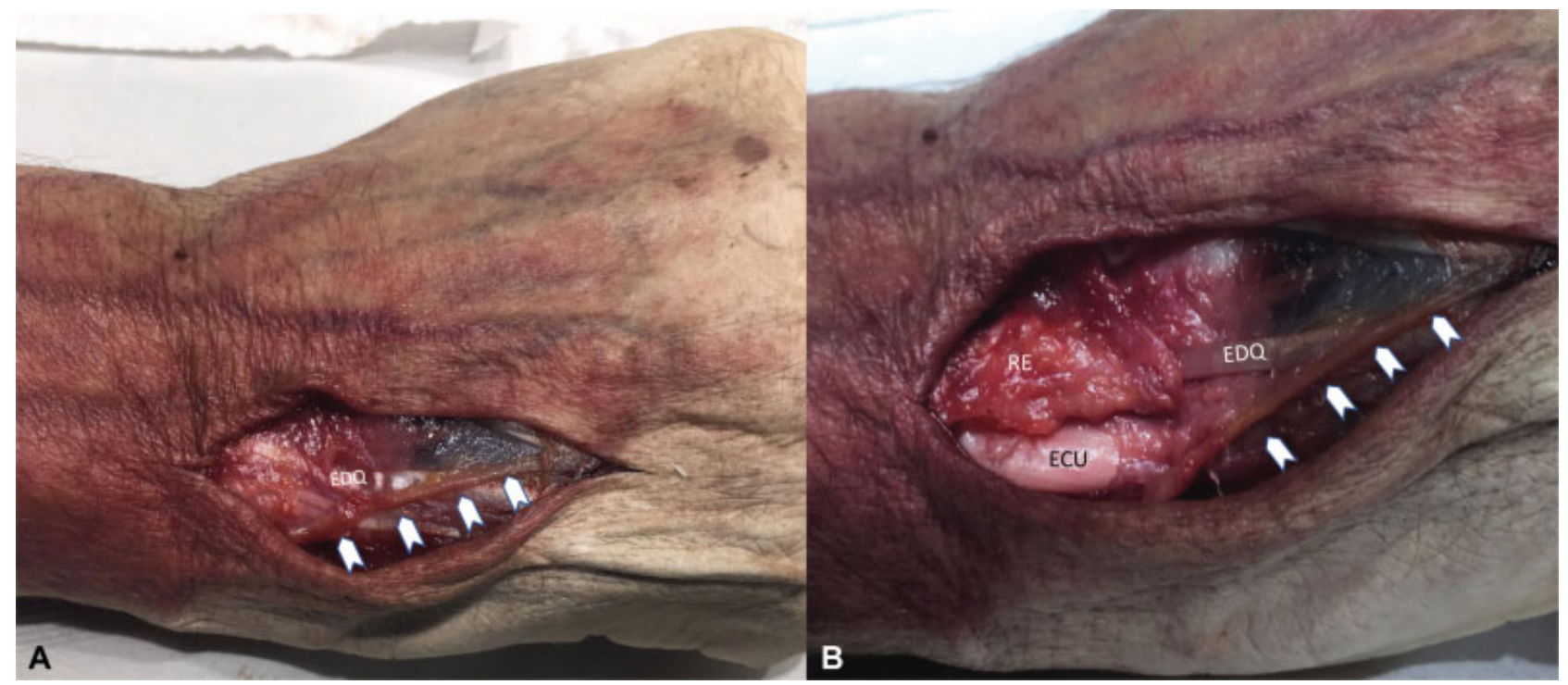

Fig. 1 (A) Incisión dorsal entre la cabeza del cúbito y la base del $5^{\circ}$ metacarpiano. Se identifica la rama sensitiva dorsal del nervio cubital (puntas de flecha blancas), que cruzan el extensor propio del quinto dedo. (B) Se abre el retináculo extensor sobre el tendón del extensor cubital del carpo. Preparación en cadáver. Abreviaciones: ECU, tendón del extensor cubital del carpo; EDQ, tendón extensor propio del $5^{\circ}$ dedo; RE, retináculo extensor.

ganchoso. La intervención se realiza bajo anestesia locoregional (plexo braquial axilar o supraclavicular, según la preferencia del anestesista), con isquemia preventiva tras la expresión de la extremidad superior con venda de Smarch, y control radioscópico. El paciente se posiciona en decúbito supino con el brazo y la mano lesionada sobre una mesa auxiliar radiotransparente para cirugía de mano.

Tras el lavado, aplicación del antiséptico y pañeado, se marca el abordaje con un rotulador dermográfico estéril.

La incisión principal es longitudinal, de unos $3,5 \mathrm{~cm}$, y se extiende entre la cabeza del cúbito y la base del $5^{\circ}$ metacarpiano. Se puede realizar una incisión accesoria, de $1 \mathrm{~cm}$, proximal a la cabeza del cúbito para que la banda tendinosa que obtengamos tenga la longitud suficiente. Después de elevar los colgajos cutáneo-grasos, la primera estructura a identificar son las ramas sensitivas dorsales provenientes del nervio cubital, que a este nivel ya están divididas, y discurren de proximal-palmar a distal-dorsal, cruzando por encima del tendón extensor propio del $5^{\circ}$ dedo (-Fig. 1). Las ramas nerviosas pueden encintarse con un "vessel-loop" fino o separarse con cuidado con la grasa subcutánea, generalmente hacia el lado medial de la

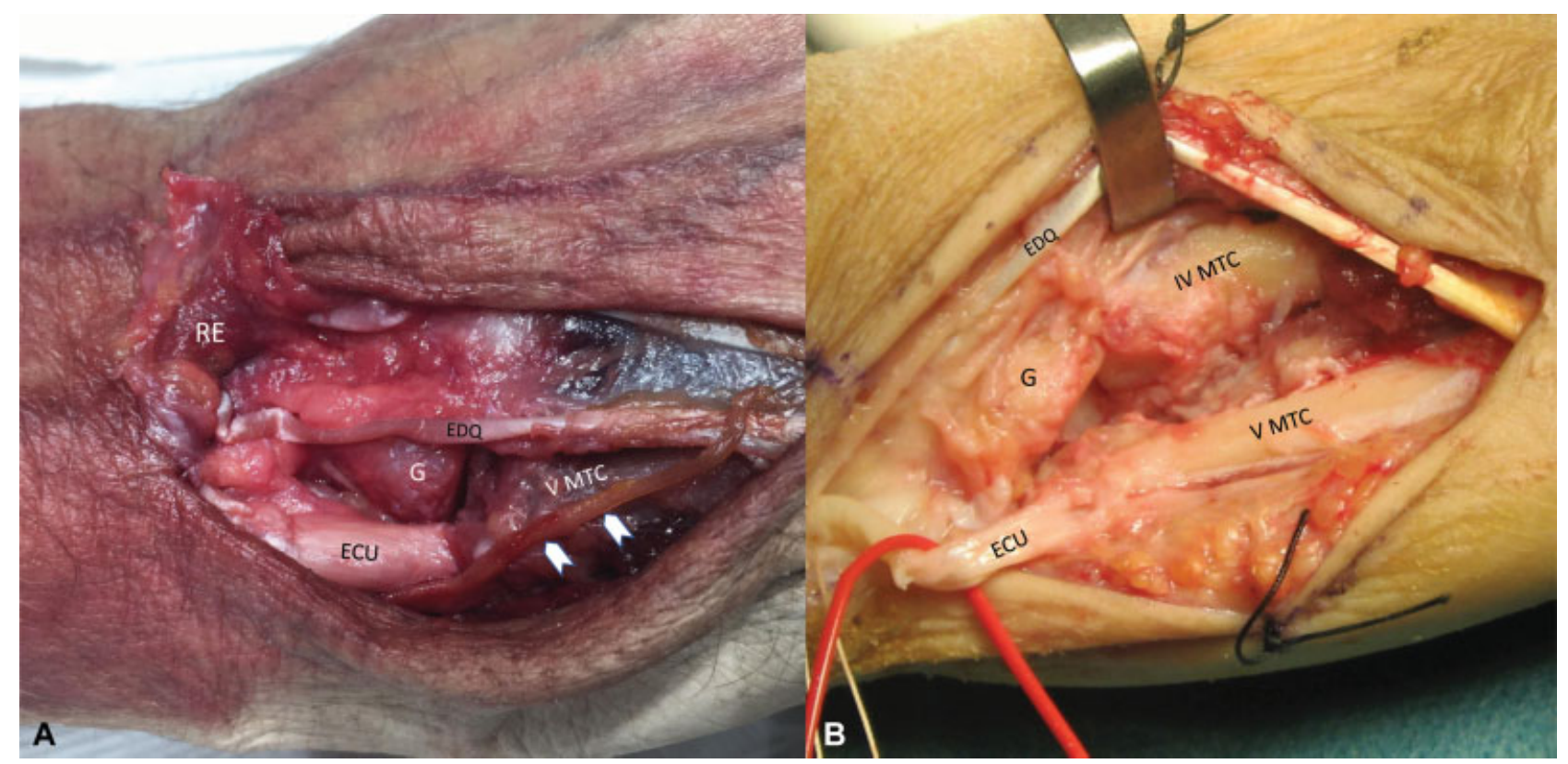

Fig. 2 (A) Capsulotomía hamatometacarpiana (preparación en cadáver). Rama sensitiva dorsal del nervio cubital (puntas de flecha blancas). (B) Desbridamiento de la articulación intermetacarpiana y hamato-metacarpiana (imagen intraoperatoria de paciente con luxación inveterada). Se señalan las mismas estructuras, pero ya la disección se extiende hasta el $4^{\circ}$ metacarpiano. Abreviaciones: ECU, tendón del extensor cubital del carpo; EDQ, tendón extensor propio del $5^{\circ}$ dedo; G, ganchoso; IVMTC, cuarto metacarpiano; RE, retináculo extensor; VMTC, quinto metacarpiano. 


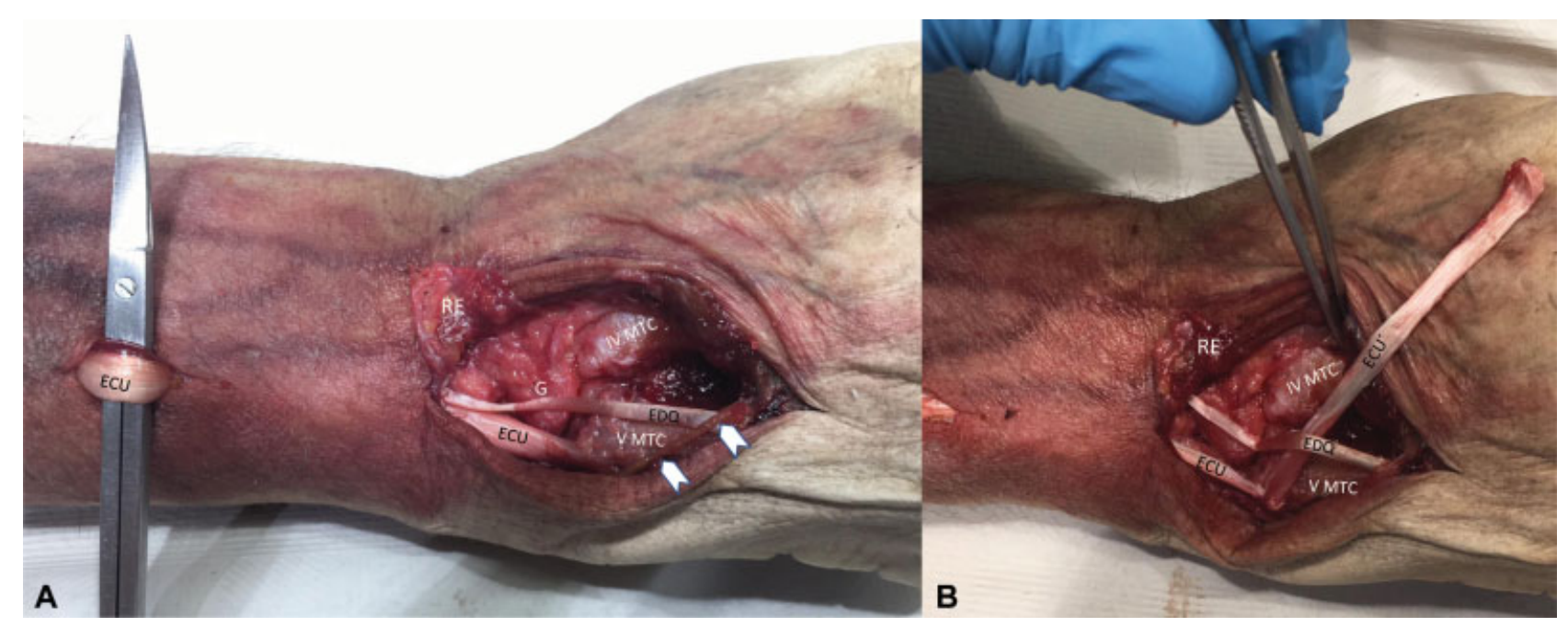

Fig. 3 (A) Mediante una incisión auxiliar proximal podemos acceder al tendón del ECU. Preparación en cadáver. (B) La banda tendinosa se exterioriza en la incisión inicial y se libera hasta su inserción distal en la base del $5^{\circ}$ metacarpiano, que se conserva. Preparación en cadáver. Abreviaciones: ECU, tendón del extensor cubital del carpo; EDQ, tendón extensor propio del $5^{\circ}$ dedo; G, ganchoso; IVMTC, cuarto metacarpiano; VMTC, quinto metacarpiano; RE, retináculo extensor.

herida. Ahora, se abre parcial o totalmente el retináculo extensor sobre el tendón del ECU $\left(6^{\circ}\right.$ compartimento extensor de la muñeca) y se eleva hasta exponer el tendón extensor propio del $5^{\circ}$ dedo ( $5^{\circ}$ compartimento extensor) [-Fig. 1]. Desplazando el tendón del extensor propio, hacia un lado u otro, es posible realizar una capsulotomía que exponga la articulación CMC del $5^{\circ}$ dedo (-Fig. 2A). Generalmente, la cápsula articular en una luxación con varias semanas de evolución, está desgarrada, desdibujada y en un lecho de fibrosis. La disección se extiende entonces en sentido distal y radial para exponer subperiosticamente la

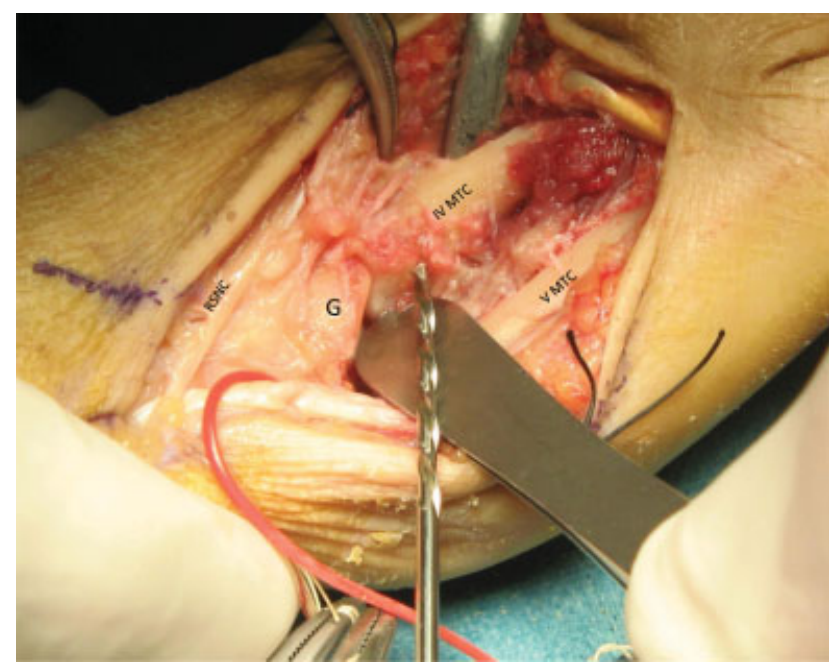

Fig. 4 El $5^{\circ}$ metacarpiano (VMTC) se desplaza en sentido palmar mediante una pinza o separador de Hohmann, para acceder al aspecto ulnar del $4^{\circ}$ (IVMTC) y realizar un túnel óseo con una broca de $3 \mathrm{~mm}$, inmediatamente distal al cartílago articular de la articulación intermetacarpiana, con una dirección ligeramente oblicua, de ulnar proximal a radial distal (imagen intraoperatoria de paciente con luxación inveterada). Abreviaciones: G, hueso ganchoso; IVMTC, cuarto metacarpiano; RSNC, rama sensitiva nervio cubital; VMTC: quinto metacarpiano. base del $4^{\circ}$ y $5^{\circ}$ metacarpiano. En este último, observamos la inserción del tendón del ECU ( - Fig. 2A y 2B).

Realizamos el desbridamiento del hematoma o de la fibrosis, dependiendo del tiempo de evolución, que impiden la reducción de la luxación y se inspeccionan las superficies articulares (-Fig. 2B).

Mediante una incisión auxiliar proximal podemos acceder al tendón del ECU, proximal al retináculo extensor ( - Fig. 3A) y tomar una tira de base distal, aproximadamente de un tercio del grosor del tendón, a unos $8 \mathrm{~cm}$ (que es la longitud recomendada) de la inserción del mismo en el metacarpiano. La banda tendinosa se exterioriza en la incisión inicial y se libera hasta su inserción distal en la base del $5^{\circ}$ metacarpiano, que se conserva ( - Fig. 3B).

La inestabilidad del $5^{\circ}$ metacarpiano, permite desplazarlo en sentido palmar mediante una pinza o separador de Hohmann, para acceder al aspecto ulnar del $4^{\circ}$ y realizar un túnel óseo con una broca de $3 \mathrm{~mm}$, inmediatamente distal al cartílago articular de la articulación intermetacarpiana, con una dirección ligeramente oblícua, de ulnar proximal a radial distal (-Fig. 4), lo más centrada posible, para evitar fractura del metacarpiano. Una medida de seguridad, es realizarlo con una broca canulada tras la colocación previa de una aguja guía en la posición y dirección deseada.

Mediante un asa de hilo de polipropileno del "0", se pasa la plastia por debajo del extensor propio del quinto dedo, por el túnel óseo y se refleja sobre sí misma (—Fig. 5). Entonces se procede a la reducción del $5^{\circ}$ metacarpiano, pero no por tracción del tendón, o se producirá una malrotación del $5^{\circ}$ dedo, pues el tendón queda en el dorso del $5^{\circ}$ metacarpiano y, sin embargo, en la parte central del $4^{\circ}$. La reducción de la articulación intermetacarpiana debe hacerse por tracción del dedo y ajustarse con unas pinzas de maleolo. Cuando visual y radiográficamente la alineación es perfecta, se realiza una fijación al $4^{\circ}$ metacarpiano y al hueso ganchoso con sendas agujas de Kirschner (- Fig. 6). Después se comprueba que se ha corregido el componente rotacional de la deformidad, 


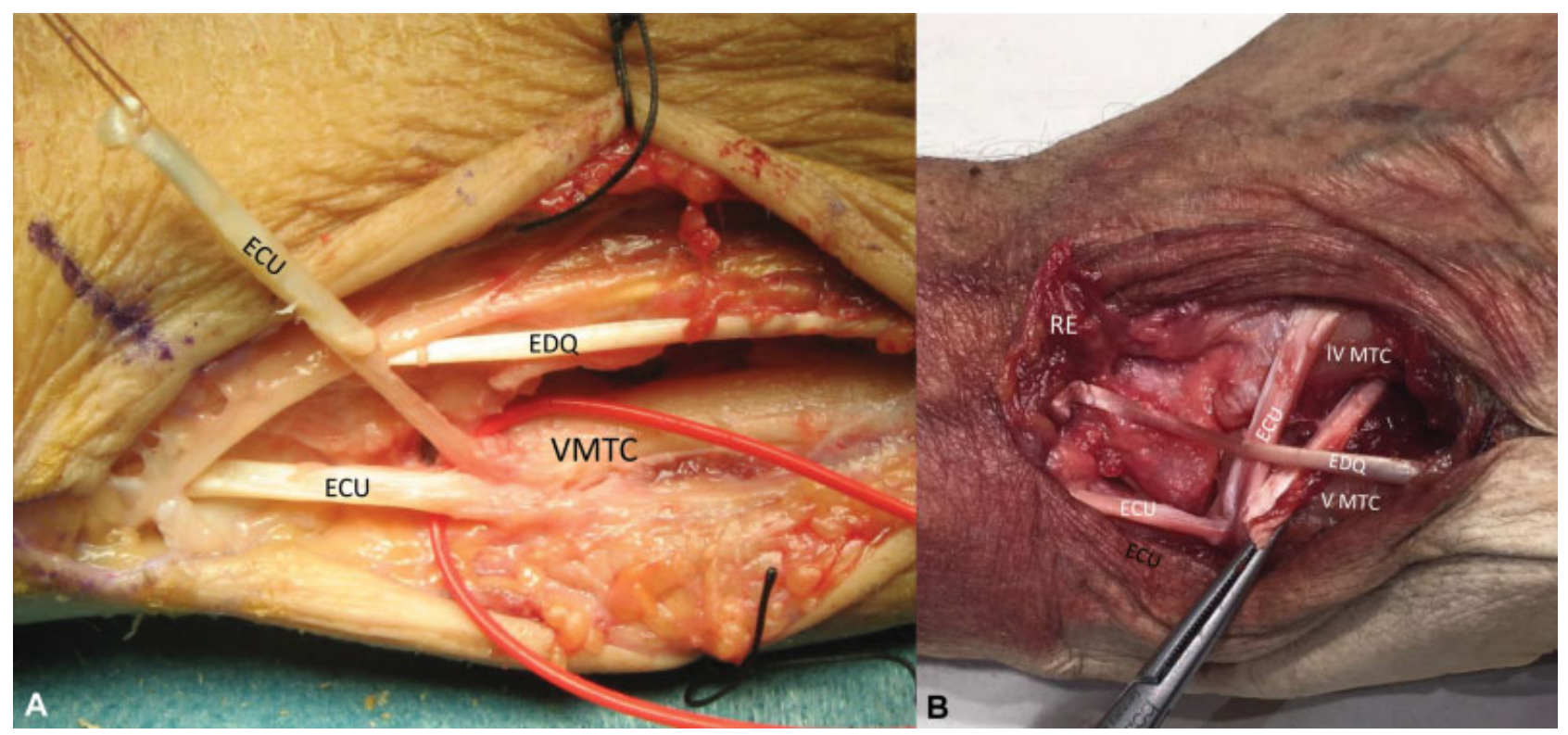

Fig. 5 (A) Exteriorización de la banda del tendón del extensor cubital del carpo en el abordaje distal (imagen intraoperatoria de paciente con luxación inveterada). (B) Paso de la misma por túnel óseo en $4^{\circ}$ metacarpiano y reflexión sobre sí (Preparación en cadáver). Abreviaciones: ECU: tendón del extensor cubital del carpo; EDQ: tendón extensor propio del $5^{\circ}$ dedo; IVMTC, cuarto metacarpiano; VMTC: quinto metacarpiano.

flexionando la articulación metacarpofalángica del $5^{\circ}$ radio y observando que todos los dedos se dirigen en paralelo hacia el tubérculo del escafoides. Solo cuando la reducción se ha fijado convenientemente, se tensa moderadamente la plastia y se sutura sobre sí mismo con poliglactina u otro material reabsorbible de $2 / 0$ o $3 / 0$. Finalmente, se procede al cierre de la herida.

El procedimiento es técnicamente sencillo, pero insistimos en que la plastia es un refuerzo de contención y no un método de reducción por tracción de la banda tendinosa a través del túnel, porque entonces se producirá una disrotación de forma sistemática. Pruzansky y cols., ${ }^{14}$ propusieron realizar un túnel óseo, no sólo en el $4^{\circ}$ metacarpiano, sino también en el $5^{\circ}$, de tal manera que la plastia cruce ambos en mitad de su eje longitudinal, y de esta forma disminuir el riesgo de disrotación.

Las principales diferencias del procedimiento que nosotros realizamos con el propuesto por Kato y Fukumoto, ${ }^{11}$ es que ellos lo indican en pacientes en fase de artrosis, realizan la escisión de la base del $5^{\circ}$ metacarpiano y la plastia con la banda del tendón del ECU no se sutura sobre sí misma, sino que se ancla a la inserción del tendón del Extensor Carpi Radialis Brevis, lo cual exige más disección.
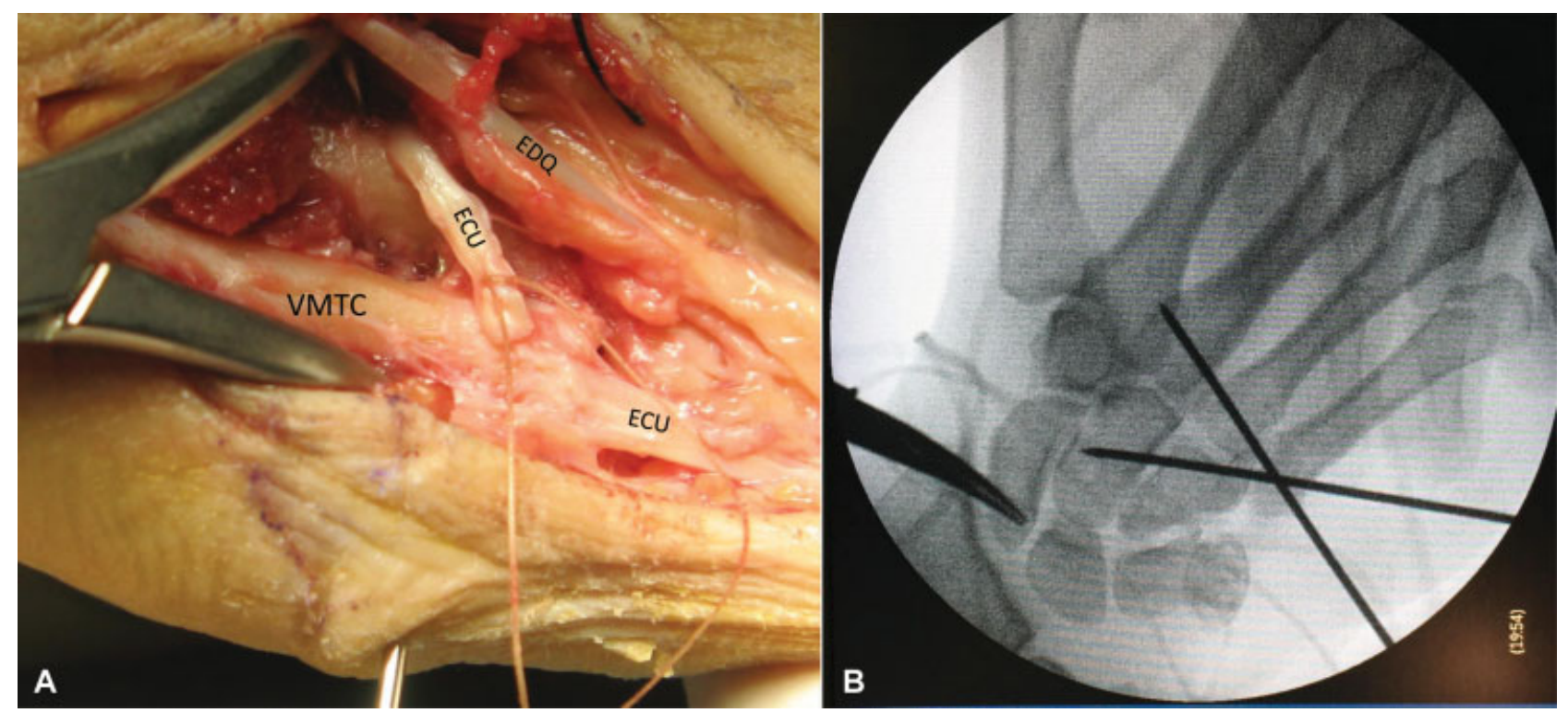

Fig. 6 (A) Reducción de la luxación con ayuda de pinzas de maleolo y sin tensar la plastia. (B) Control radioscópico de la fijación de la reducción con Agujas de Kirschner. Abreviaciones: ECU: tendón del extensor cubital del carpo; EDQ: tendón extensor propio del $5^{\circ}$ dedo; VMTC: quinto metacarpiano. 


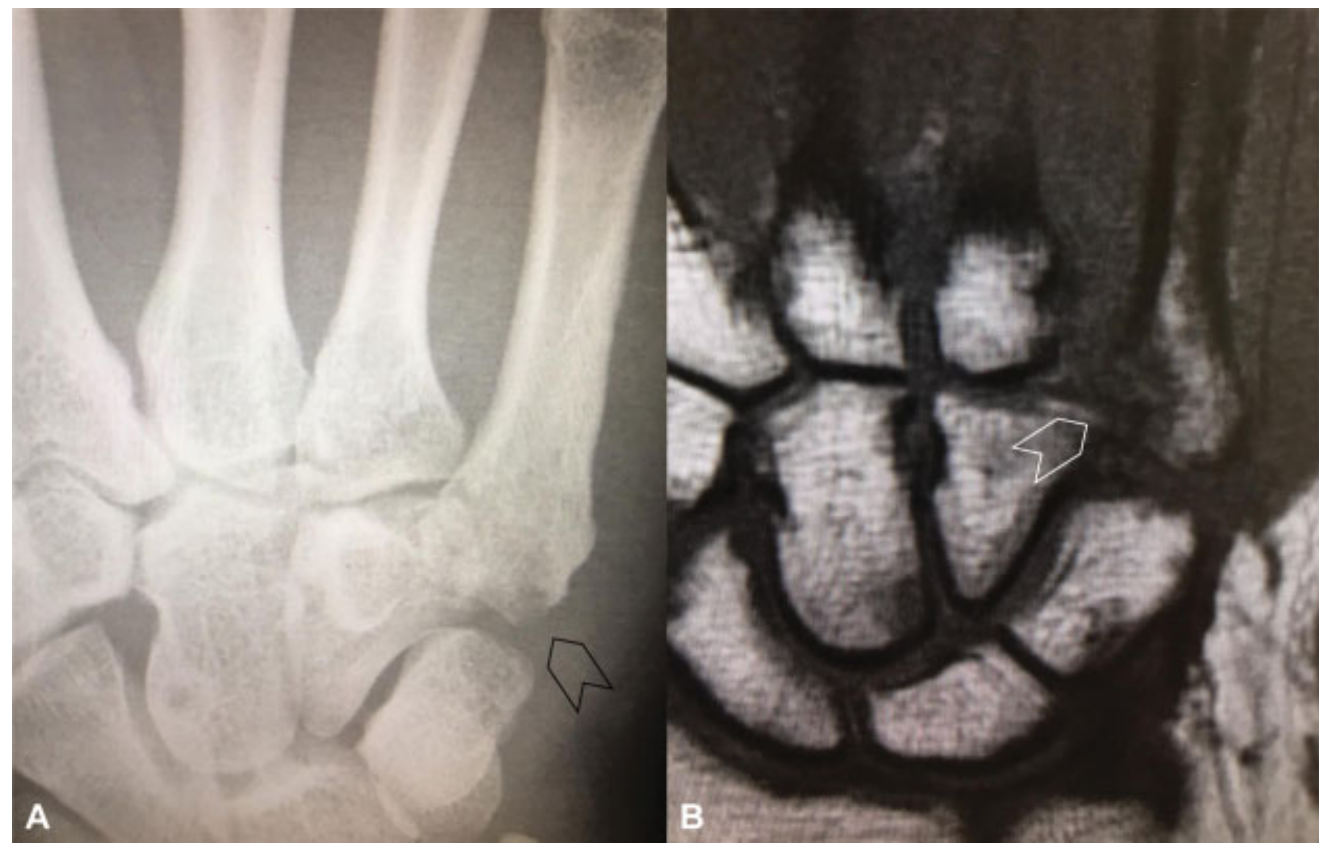

Fig. 7 (A) Radiografía y (B) RNM de la mano del paciente del caso clínico. La punta de flecha señala la luxación CMC del $5^{\circ}$ radio.

\section{Postoperatorio}

Habitualmente, no dejamos drenaje. El paciente se da de alta en 24 horas, tras el primer control radiográfico. Mantenemos una férula antebraquial corta que deja libre la articulación metacarpo-falángica y se permite la movilidad digital desde el primer día. La férula y la agujas de Kirschner se retiran en consulta después de las 4-5 semanas. Se solicita inmediatamente después un control radiográfico y se propone una revisión 2-3 semanas después con nueva radiografía para valorar un posible desplazamiento secundario, y si está afectada la movilidad, se instaura tratamiento rehabilitador. La actividad manual exigente se permite, siempre de forma progresiva, a partir de los 3 meses.

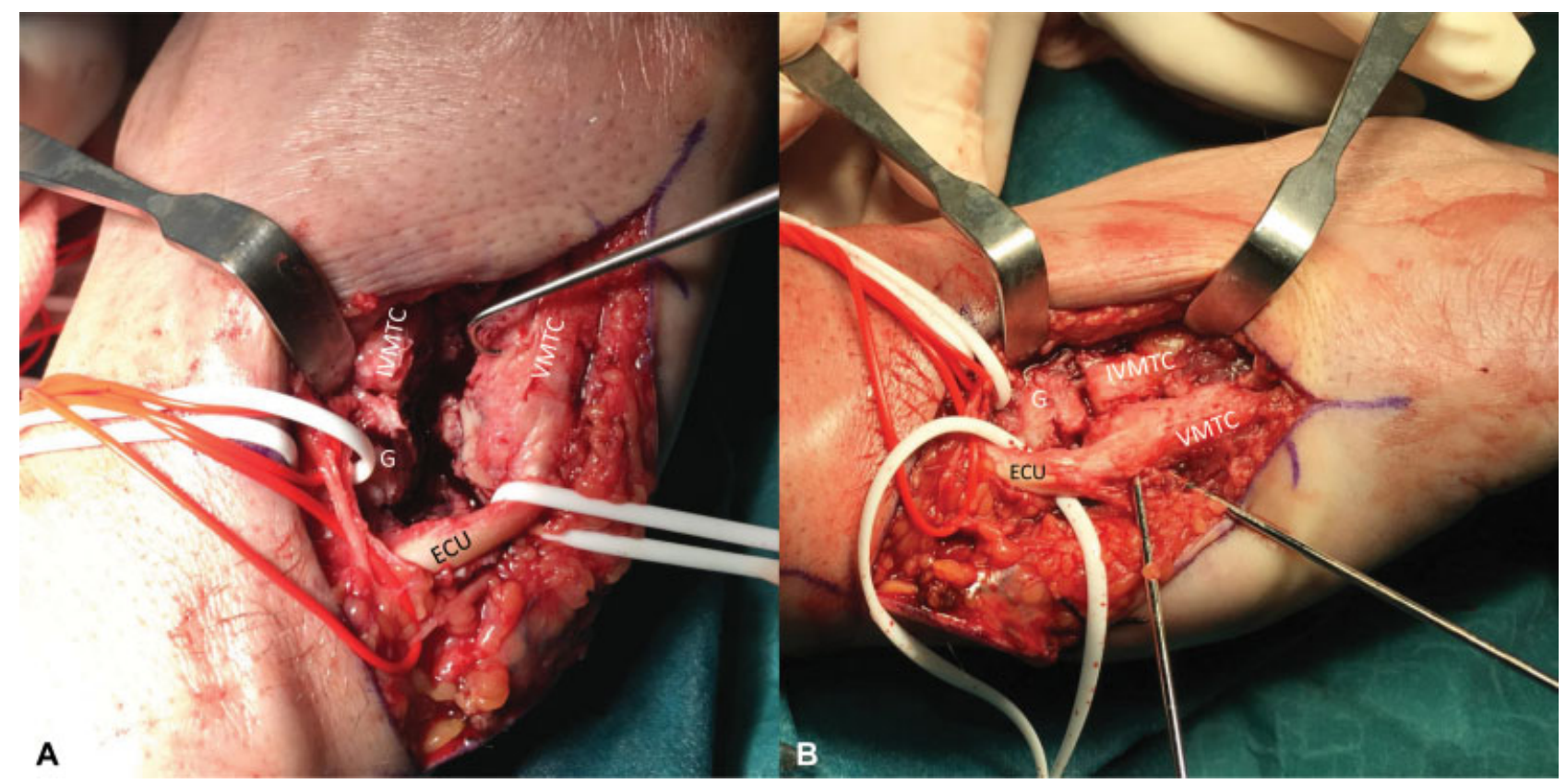

Fig. 8 (A) Exposición quirúrgica de luxación $C M C$ del $5^{\circ}$ radio de 4 semanas de evolución. Se observa la disociación entre $4^{\circ}$ y $5^{\circ}$ metacarpiano, así como la migración proximal y ulnar del último respecto al hueso ganchoso, bajo la tracción del tendón del extensor cubital del carpo. (B) Reducción y fijación con dos agujas de Kirschner, después de haber realizado el túnel óseo en el $4^{\circ}$ metacarpiano. Abreviaciones: ECU: tendón del extensor cubital del carpo; G, hueso ganchoso; IVMTC, cuarto metacarpiano; VMTC: quinto metacarpiano. 


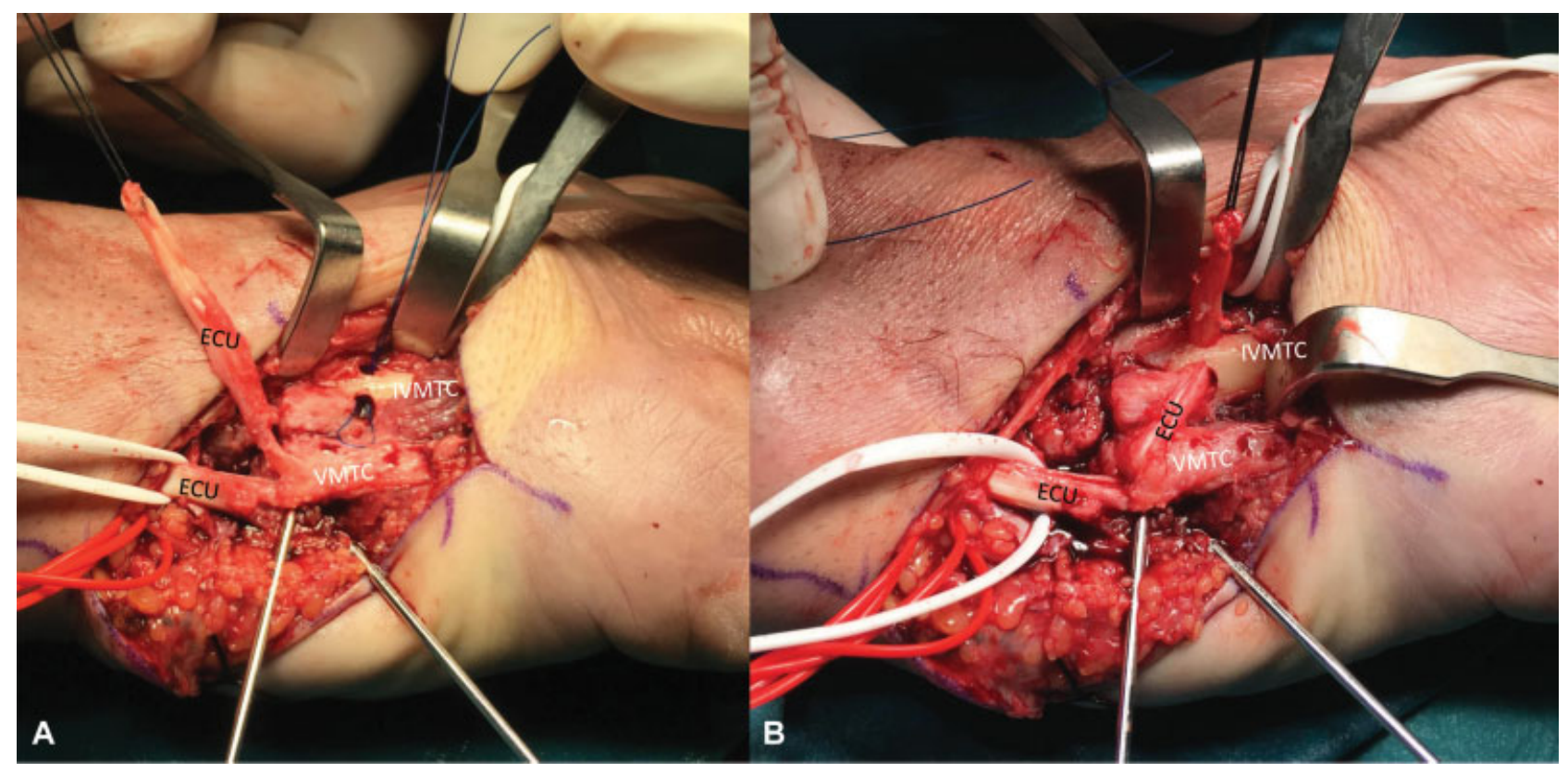

Fig. 9 (A) Toma de la banda del tendón del extensor cubital del carpo para plastia, respetando su inserción distal. En el $4^{\circ}$ metacarpiano se observa el orificio ulnar del túnel óseo. Pasamos una sutura de polipropileno para recuperar la plastia. (B) En esta visión, la banda de tendón ya ha atravesado el túnel óseo. Abreviaciones: ECU: tendón del extensor cubital del carpo; IVMTC, cuarto metacarpiano; VMTC: quinto metacarpiano.

\section{Complicaciones}

Las complicaciones posibles son la infección de la herida quirúrgica, la lesión de la rama sensitiva dorsal del nervio cubital y el desarrollo de un neuroma, la rigidez digital por adherencia de los extensores en el lecho quirúrgico, rara vez la sección del tendón extensor propio del $5^{\circ}$ dedo, la luxación del tendón del ECU, la fractura intraoperatoria del $4^{\circ}$ metacarpiano, la deformidad en malrotación o acortamiento del $5^{\circ}$ dedo por perdida de reducción o reducción inadecuada inicial, y a largo plazo, el desarrollo de una artrosis postraumática de la articulación CMC.

\section{Caso Clínico}

Paciente varón de 46 años, que sufre traumatismo de su mano derecha al caer de un caballo. Consultó por Urgencias debido a dolor, deformidad, disrotación del $5^{\circ}$ dedo y dificultad para cerrar la mano. Fue diagnosticado de contusión y posteriormente, remitido por el médico de atención primaria a nuestra consulta, 4 semanas después, por mala evolución, con la radiografía y RNM que se observa en la - Figura 7. Fue entonces diagnosticado de luxación CMC del $5^{\circ}$ dedo y se propuso tratamiento quirúrgico. Se realizó reducción abierta, tenosuspensión por la técnica detallada y

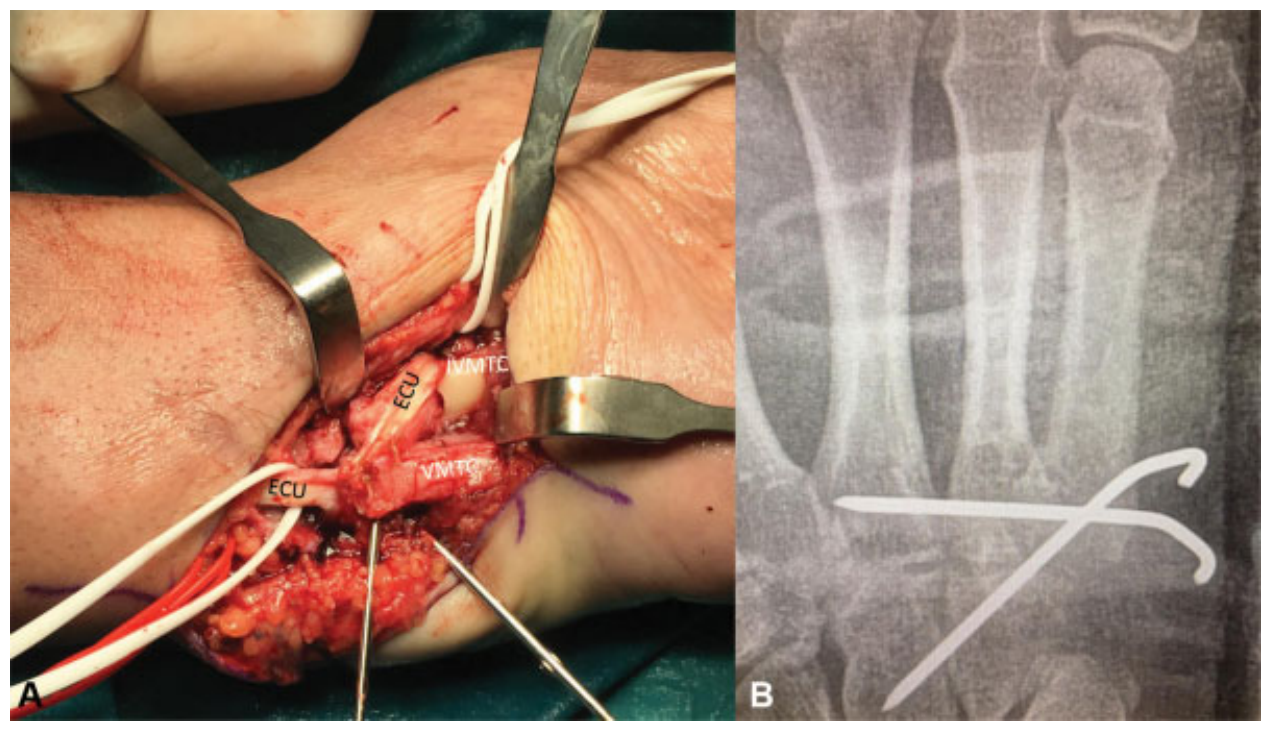

Fig. 10 (A) Aspecto final de la reparación, con la tenosuspensión concluida al suturar la banda tendinosa reflejada a la inserción del extensor cubital del carpo en la base del $5^{\circ}$ metacarpiano. (B) Control radiográfico postoperatorio inmediato. Abreviaciones: ECU: tendón del extensor cubital del carpo; IVMTC, cuarto metacarpiano; VMTC: quinto metacarpiano. 


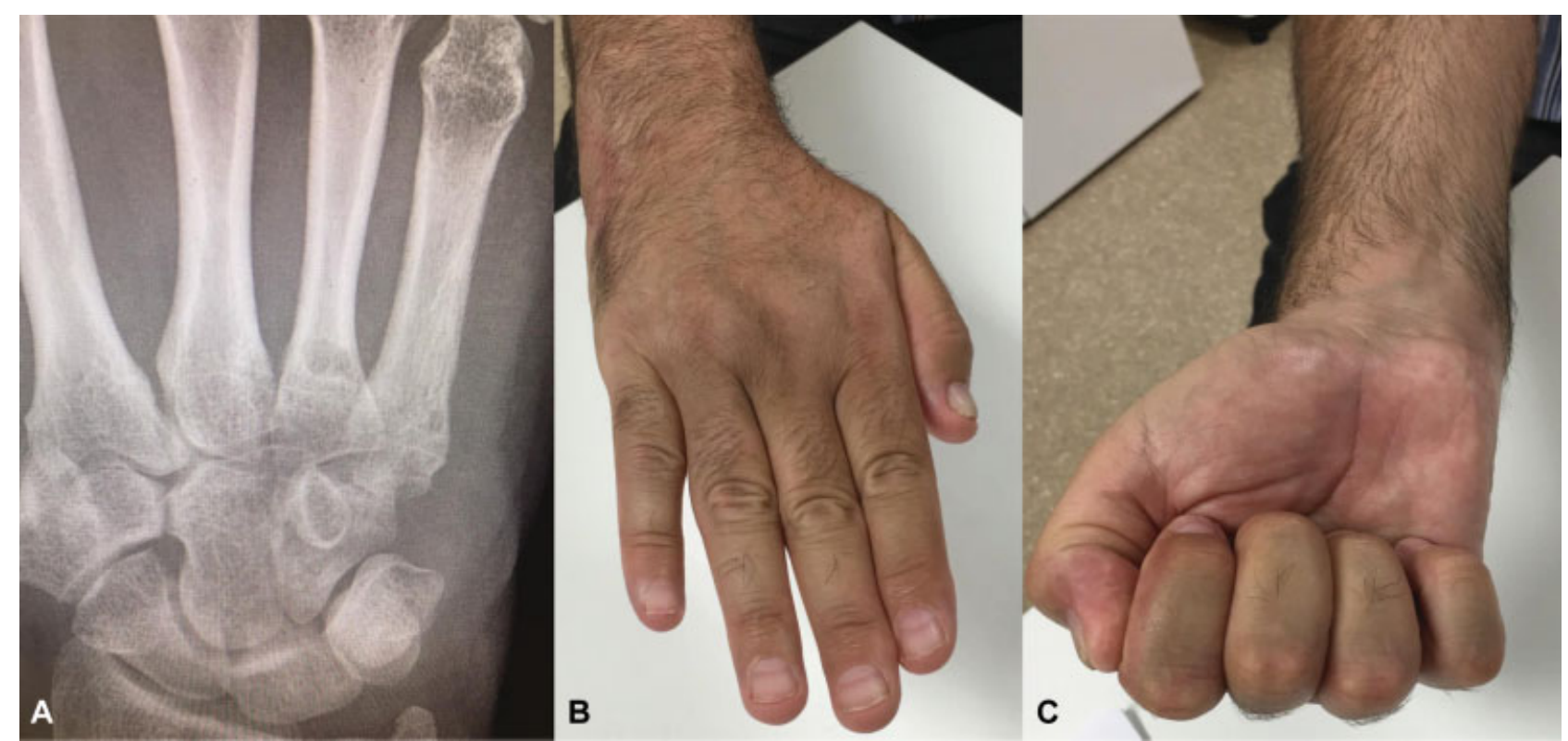

Fig. 11 (A) Control radiográfico 2 años después de la intervención. (B y C) Resultado funcional de la prensión del paciente.

fijación provisional con agujas de Kirschner (-Figs. 8-10). Dos años después, la radiografía mantiene la reducción CMC sin signos degenerativos (-Fig. 11) y el resultado funcional es satisfactorio para el paciente.

Conflictos de interés

Ninguno.

\section{Bibliografía}

1 McDonald LS, Shupe PG, Hammel N, Kroonen LT. The intermetacarpal angle screening test for ulnar-sided carpometacarpal fracturedislocations. J Hand Surg Am 2012;37(09):1839-1844

2 Kim JK, Shin SJ. A novel hamatometacarpal fracture-dislocation classification system based on CT scan. Injury 2012;43(07): 1112-1117

3 Cobb WA, Dingle L, Zarb Adami R, Rodrigues J. Management of fracture-dislocations of the little finger carpometacarpal joint: a systematic review. J Hand Surg Eur Vol 2018;43(05):530-538

4 Zhang C, Wang H, Liang C, et al. The Effect of Timing on the Treatment and Outcome of Combined Fourth and Fifth Carpometacarpal Fracture Dislocations. J Hand Surg Am 2015;40(11):2169-2175.e1

5 Bao B, Zhu H, Zheng X. Fourth and fifth carpometacarpal fracturedislocations is plate or Kirschner wire fixation superior: A retrospective cohort study. Int J Surg 2018;52:293-296
6 Hunt TR III. Degenerative and post-traumatic arthritis affecting the carpometacarpal joints of the fingers. Hand Clin 2006;22(02): 221-228

7 Clendenin MB, Smith RJ. Fifth metacarpal/hamate arthrodesis for posttraumatic osteoarthritis. J Hand Surg Am 1984;9(03):374-378

8 Dubert TP, Khalifa H. "Stabilized arthroplasty" for old fracture dislocations of the fifth carpometacarpal joint. Tech Hand Up Extrem Surg 2009;13(03):134-136

9 Black DM, Watson HK, Vender MI. Arthroplasty of the ulnar carpometacarpal joints. J Hand Surg Am 1987;12(06): 1071-1074

10 Green WL, Kilgore ES Jr. Treatment of fifth digit carpometacarpal arthritis with Silastic prosthesis. J Hand Surg Am 1981;6(05): 510-514

11 Kato N, Fukumoto K. Suspension Arthroplasty for Old FractureDislocations of the Fifth Carpometacarpal Joint. Tech Hand Up Extrem Surg 2016;20(02):83-87

12 Bushnell BD, Draeger RW, Crosby CG, Bynum DK. Management of intra-articular metacarpal base fractures of the second through fifth metacarpals. J Hand Surg Am 2008;33(04):573-583

13 Syed AA, Agarwal M, Giannoudis PV, Matthews SJE. Dorsal hamatometacarpal fracture-dislocation in a gymnast. $\mathrm{Br} \mathrm{J}$ Sports Med 2002;36(05):380-382

14 Pruzansky JS, Goljan P, Bachoura A, Jacoby SM, Culp RW. Little finger carpometacarpal arthroplasty technique and result in 3 cases. J Hand Surg Am 2014;39(09):1734-1738 